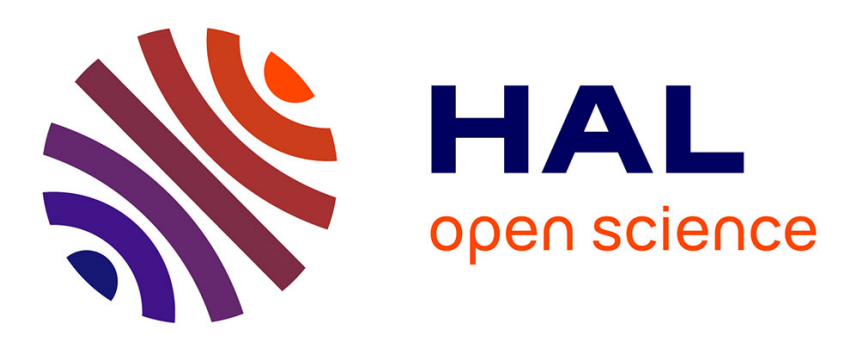

\title{
Analysis vs Synthesis-based Regularization for combined Compressed Sensing and Parallel MRI Reconstruction at 7 Tesla
}

H. Cherkaoui, L. El Gueddari, C. Lazarus, A. Grigis, F. Poupon, A. Vignaud, S. Farrens, J.-L. Starck, Philippe Ciuciu

\section{To cite this version:}

H. Cherkaoui, L. El Gueddari, C. Lazarus, A. Grigis, F. Poupon, et al.. Analysis vs Synthesis-based Regularization for combined Compressed Sensing and Parallel MRI Reconstruction at 7 Tesla. 26th European Signal Processing Conference (EUSIPCO 2018), Sep 2018, Roma, Italy. hal-01800700v2

\section{HAL Id: hal-01800700 \\ https://hal.inria.fr/hal-01800700v2}

Submitted on 18 Jun 2018

HAL is a multi-disciplinary open access archive for the deposit and dissemination of scientific research documents, whether they are published or not. The documents may come from teaching and research institutions in France or abroad, or from public or private research centers.
L'archive ouverte pluridisciplinaire HAL, est destinée au dépôt et à la diffusion de documents scientifiques de niveau recherche, publiés ou non, émanant des établissements d'enseignement et de recherche français ou étrangers, des laboratoires publics ou privés. 


\title{
Analysis vs Synthesis-based Regularization for combined Compressed Sensing and Parallel MRI Reconstruction at 7 Tesla
}

\author{
H. Cherkaoui, L. El Gueddari, C. Lazarus, A. Grigis, F. Poupon, A. Vignaud, S. Farrens, J.-L. Starck, P. Ciuciu \\ CEA Saclay, Univ. Paris-Saclay, 91191 Gif-sur-Yvette, France. \\ firstname. lastnamedcea.fr
}

\begin{abstract}
Compressed Sensing (CS) has allowed a significant reduction of acquisition times in MRI, especially in the high spatial resolution (e.g., $400 \mu \mathrm{m})$ context. Nonlinear CS reconstruction usually relies on analysis (e.g., Total Variation) or synthesis (e.g., wavelet) based priors and $\ell_{1}$ regularization to promote sparsity in the transform domain. Here, we compare the performance of several orthogonal wavelet transforms with those of tight frames for MR image reconstruction in the CS setting combined with parallel imaging (multiple receiver coil). We show that overcomplete dictionaries such as the fast curvelet transform provide improved image quality as compared to orthogonal transforms. For doing so, we rely on an analysis-based formulation where the underlying $\ell_{1}$ regularized criterion is minimized using a primal dual splitting method (e.g., Condat-Vũ algorithm). Validation is performed on ex-vivo baboon brain $T_{2}^{*}$ MRI data collected at 7 Tesla and restrospectively under-sampled using non-Cartesian schemes (radial and Sparkling). We show that multiscale analysis priors based on tight frames instead of orthogonal transforms achieve better image quality (pSNR, SSIM) in particular at low signal-to-noise ratio.
\end{abstract}

\section{INTRODUCTION}

Context: Compressed-Sensing MRI. Magnetic Resonance Imaging (MRI) is a key imaging technique to probe soft tissues (e.g., the brain) non-invasively. However, its acquisition time may be prohibitive in the context of high resolution imaging. To cope with this issue, several approaches such as parallel imaging (PI) [1], [2] or Compressed Sensing (CS) [3], [4] have been developed and successively applied to MRI [5] for more than 10 years. PI usually permits to accelerate MRI scans by a 6-8 factor but at the expense of a degraded signal-tonoise ratio (SNR) owing to deterministic under-sampling and aliasing artifacts that arise between channels of the phasedarray receiver coil. Instead, CS enables to accelerate MRI exams by randomly under-sampling the data collected in the Fourier domain ( $k$-space) while providing theoretical guarantees of exact image recovery [3]-[5]. This theory is based on (i) sparsity or compressibility of MR images in a given dictionary (e.g., wavelets), (ii) incoherence between sensing and sparsity bases and (iii) nonlinear image reconstruction for promoting sparsity in the transform domain.

Over the last decade, several approaches have been proposed for combining CS and parallel MRI reconstruction either using (possibly joint) sparsity or low-rank regularizing constraints, cf e.g. [6]-[12]. Here, in the combined CS-PI setting, we focus on sparsity promoting regularization which is usually enforced using the $\ell_{1}$-norm of an analysis or a synthesis-based prior. The analysis-based prior operates in the image domain and involves some sort of derivative operators (e.g., Total Variation), whereas the synthesis prior acts in the transform domain for sparse multiscale image representation, such as the wavelet transform (WT).

Related works: Analysis vs Synthesis penalties in MRI. This topic has been intensively covered in the literature over the last decade by comparing the theoretical properties and practical performance of analysis and synthesis priors in the context of undercomplete, complete and overcomplete transforms [13], [14]. Importantly, it has been shown that in the simpler complete (e.g., orthogonal WT) formulations, analysis and synthesis based formulations are equivalent, whereas they depart one another in their overcomplete formulation (e.g., curvelet, contourlet). Interestingly, it has been shown that the analysis-based formulation provides more accurate results in the overcomplete case, i.e. when redundant dictionaries are used for regularization, cf e.g. [14]. In image analysis and more specifically for MRI reconstruction, the use of undecimated wavelet transforms permits to guarantee the translation invariance of the image solution [8]. For improved localization properties within the space domain and excellent directional selectivity, several groups have tested discrete nonseparable shearlet transforms [15]. Although shearlets are known to be an optimal multiscale sparse representation for natural images, they suffer from high computational cost. Yet, the fast curvelet transform [16], [17] is an efficient and competitive alternative that is endowed with the same approximation rate than shearlets but that has not been tested so far for MR image reconstruction from highly under-sampled $k$-space real data.

Goals and contributions. We first recall the analysis vs synthesis-based formulation of the CS parallel MRI reconstruction problem in Section II, the analysis one being the convenient approach for dealing with overcomplete dictionaries and synthesis one for orthogonal transforms. In the analysis case, a primal dual splitting method, known as the CondatVũ algorithm [18], [19] is implemented. Alternatively, for the synthesis-based formulation, an Nesterov accelerated proximal gradient method (e.g., FISTA algorithm) is used [20], [21]. Ex-vivo baboon brain high resolution 2D MRI data collected at 7 Tesla and retrospectively under-sampled using different 
$k$-space trajectories and various under-sampling factors are presented in Section IV. In Section V, we propose an quantitative comparison of the performances of various multiscale decompositions (orthogonal Mallat WT, Meyer WT, B-spline WT, undecimated bi-orthogonal WT and fast curvelets) for combined CS-PI MR image reconstruction. MR image quality are compared in terms of peak SNR (pSNR) and structural similarity (SSIM) metrics across all tested transforms and for various input SNR and under-sampling schemes. We choose those various multiscale decompositions to represent previous choice done in the MRI image reconstruction field. We can distinguish three mains categories from the litterature: the orthogonal wavelet basis (e.g. Daubechie wavelet), tight frame (e.g. Ridgelet) and representation that sparsely encode geometrical properties like a sparse representation of curvature (e.g. Curvelet). Note that the fast curvelet transform has never been used for an MRI reconstruction problem. In short, in this paper, we show that tight frames provide better performances in terms of image quality, especially at low input SNR. Conclusions are drawn in Section VI.

\section{CS-PI MRI RECONSTRUCTION}

Let $L$ be the number of coils used to acquire the NMR signal, $N$ be the number of pixels of the complex-valued image $\boldsymbol{x}$ to be reconstructed and $M$ the number of samples collected per channel during acquisition. We denote by $\boldsymbol{y}_{\ell} \in \mathbb{C}^{M}$ the complex-valued data recorded by the $\ell^{\text {th }}$ channel, $\boldsymbol{S}_{\ell} \in \mathbb{C}^{N \times N}$ the corresponding diagonal sensitivity matrix. Let $F$ be the NFFT and $\Omega \in\{1, \ldots, N\}$ the sampling pattern in the $k$ space, with $|\Omega|=M \ll N$. The CS-PI acquisition model thus reads: $\forall \ell=1: L, \boldsymbol{y}_{\ell}=F_{\Omega} \boldsymbol{S}_{\ell} \boldsymbol{x}+\boldsymbol{b}_{\ell}$ where $\boldsymbol{b}_{\ell}$ is additive zero-mean Gaussian noise of variance $\sigma_{\ell}^{2}$, which can be characterized by a separate scan (without RF pulse) considering the same bandwidth as the prospective CS acquisition.

Analysis-based formulation. The unknown MR image $\boldsymbol{x} \in$ $\mathbb{C}^{N}$ is sparse in the transform domain: $\boldsymbol{\alpha}=\boldsymbol{\Psi} \boldsymbol{x}$, hence $s \triangleq \#\left\{i \in\{1, \ldots, N\}, \alpha_{i} \neq 0\right\} \ll N$. Dictionary $\boldsymbol{\Psi}$ may refer to an orthogonal wavelet WT (Daubechies or Meyer) or to a tight frame (undecimated bi-orthogonal, B-spline àtrous wavelet transform or curvelet). Hereafter, we will adopt the notation $\boldsymbol{\Psi}_{\mathrm{OT}}$ and $\boldsymbol{\Psi}_{\mathrm{TF}}$ to disentangle orthogonal transforms (OT) from tight frames (TF), with $\boldsymbol{\alpha}=\boldsymbol{\Psi}_{T F} \boldsymbol{x} \in \mathbb{C}^{P}$ and $P \gg N$. The CS-PI reconstruction problem amounts to minimizing of the $\ell_{1}$-analysis-based regularized criterion:

$$
\widehat{\boldsymbol{x}}=\underset{\boldsymbol{x} \in \mathbb{C}^{N}}{\arg \min }\left\{\sum_{\ell=1}^{L} \frac{1}{2 \sigma_{\ell}^{2}}\left\|F_{\Omega} \boldsymbol{S}_{\ell} \boldsymbol{x}-\boldsymbol{y}_{\ell}\right\|_{2}^{2}+\lambda\|\boldsymbol{\Psi} \boldsymbol{x}\|_{1}\right\},
$$

where $\lambda>0$ is the regularization parameter. This formulation requires the knowledge of the sensitivity maps $\left\{\boldsymbol{S}_{\ell}\right\}_{\ell}$. Here, we assume these maps known since they can be estimated using self-calibration techniques [22], [23].

When the dictionary $\boldsymbol{\Psi}_{T F}$ is overcomplete, the analysis formulation (1) is the most appealing because the dimension of the optimization problem is smaller. In Section III, we resort to a primal-dual splitting method to minimize Eq. (1).
Synthesis-based formulation. When $\Psi_{\mathrm{OT}}$ is orthogonal, the synthesis-based $\ell_{1}$-penalty can be injected after the change of variable $\boldsymbol{\alpha}=\boldsymbol{\Psi}_{\mathrm{OT}} \boldsymbol{x}$. The regularized criterion now reads in the transform domain:

$$
\widehat{\boldsymbol{\alpha}}=\underset{\boldsymbol{\alpha} \in \mathbb{C}^{N}}{\arg \min }\left\{\sum_{\ell=1}^{L} \frac{1}{2 \sigma_{\ell}^{2}}\left\|F_{\Omega} \boldsymbol{S}_{\ell} \boldsymbol{\Psi}_{\mathrm{OT}}^{*} \boldsymbol{\alpha}-\boldsymbol{y}_{\ell}\right\|_{2}^{2}+\lambda\|\boldsymbol{\alpha}\|_{1}\right\} .
$$

The MR image solution is then reconstructed as follows: $\widehat{\boldsymbol{x}}=$ $\Psi_{\text {OT }}^{*} \widehat{\boldsymbol{\alpha}}$. In Section III, we resort to an accelerated proximal gradient methods [20] to minimize Eq. (2).

\section{OPTIMIZATION ALGORITHMS}

\section{A. Primal-dual splitting algorithm}

The Condat-Vũ algorithm [18], [19] allows us to solve Eq. (1). Our implementation is available in Pysap (i.e., Python Sparse data Analysis Package) ${ }^{1}$. In Eq. (1), the data consistency $\ell_{2}$-norm term is a smooth convex function with a $\beta$-Lipschitz continuous gradient term that reads:

$$
\nabla f(x)=\sum_{\ell=1}^{L} \sigma_{\ell}^{-2} \boldsymbol{S}_{\ell}^{\dagger} F_{\Omega}^{\star}\left(F_{\Omega} \boldsymbol{S}_{\ell} \boldsymbol{x}-\boldsymbol{y}_{\ell}\right)
$$

where ${ }^{\dagger}$ denotes the Hermitian operator and $F^{\star}$ the conjugate of the NFFT. Importantly, because of non-Cartesian undersampling schemes (i.e., $\Omega$ is not defined on the Cartesian grid in $k$-space), the Lipschitz constant $\beta$ was computed using a power iteration method (eigenvalue decomposition). Weak convergence is guaranteed according to [19, Theorem 3.1].

\section{B. Proximal gradient methods}

The original Forward Backward (FB) algorithm allows us to solve Eq. (2). It is a generalization of gradient descent methods to non-differentiable functions, which can be expressed as follows: $x_{k+1}=\operatorname{prox}_{t_{k}}\left(x_{k}-\tau_{k} \nabla f\left(x_{k}\right)\right)$. In the specific case of the FISTA [21], the acceleration term $\tau_{k}$ is defined by: $\tau_{k+1}=\frac{1+\sqrt{1+4 \tau_{k}^{2}}}{2}$ and $\tau_{0}=1$.

\section{MATERIALS AND METHODS}

\section{A. MRI data}

For validation purposes, we have collected a single ex-vivo Cartesian baboon brain $T_{2}^{*}$-weighted MRI data at 7T (Magnetom Siemens scanner, Erlangen, Germany) using the 32channel (Nova Medical Inc., Washington, MA, USA) coil. The acquisition parameters were set as follows: $\mathrm{TR}=550 \mathrm{~ms}$, $\mathrm{TE}=30 \mathrm{~ms}$ and $\mathrm{FA}=25^{\circ}$ with an in-plane resolution of $400 \mu \mathrm{m}$ corresponding to an image matrix size of $N=$ $512 \times 512$ and a total acquisition time of $4 \min 42 \mathrm{~s}$ for 11 slices. Slice thickness was set to $3 \mathrm{~mm}$ to maintain a high SNR during acquisition. We retained only one slice from this $3 \mathrm{D}$ reference dataset for the experimentation, the chosen slice is shown in Fig. 4

From this slice, retrospective varianle density undersampling [24] was performed according to radial spokes and brand new multi-shot Sparkling trajectories [25]. Sparkling

\footnotetext{
${ }^{1}$ cf https://github.com/CEA-COSMIC/pysap
} 
shots were generated all together using the algorithm proposed in [26] to draw samples according to a variable density with a polynomial decay: $h\left(k_{x}, k_{y}\right)=1 /|k|^{2}$. These two kinds of sampling patterns are shown in Fig. 1. Two undersampling factors $R=N / m$, defined as the number of image pixels over the number of measurements, have been tested: the value $R=2.4$ and $R=3.3$ corresponding to $40 \%$ and $30 \%$ of samples respectively were used to assess the robustness of MRI reconstruction under various settings. Also, zero-mean white Gaussian noise was added to the $k$-space data for increasing variances corresponding to input SNR (ISNR) varying from $3 \mathrm{~dB}$ to $40 \mathrm{~dB}$ (noise-free) approxixmately. This allows us to mimic varying SNR due to the acquisiton of thinner slices: the ISNR in MRI is actually proportional to the voxel size and thus to the slice thickness for a fixed plane resolution. (a) Sparkling

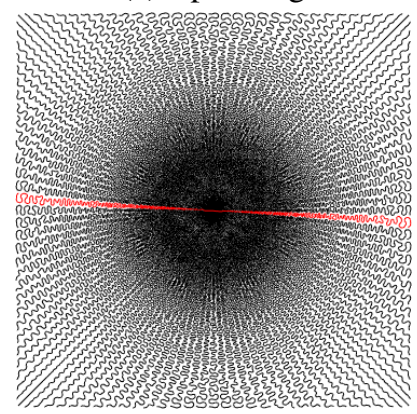

(b) Radial

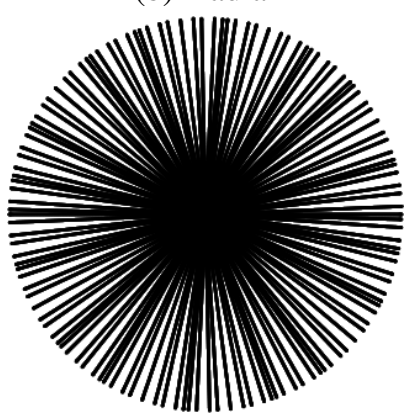

Fig. 1. Non-Cartesian multi-shot under-sampling schemes for $R=2.4$ i.e., $40 \%$ of full- $k$-space measurements.

\section{B. Methods}

We performed MR image reconstruction from the retrospectively undersampled $k$-space data collected using either the radial or Sparkling schemes over the $L=32$ channel coils. In the analysis-based formulation (1), we run the Condat-Vũ algorithm for 200 iterations to guarantee that convergence was achieved. In the synthesis-based formulation (2), we run FISTA algorithm for 200 iterations too. We swept several user-defined parameters such as the number of scales $(J \in\{3,4,5\})$ in WT and the regularization parameter $\lambda \in\left\{10^{-1}, \ldots, 10^{-8}\right\}$ involved in Eqs. (1)-(2). For each ISNR value, a grid search over 20 values of $\lambda$ and all above mentioned $J$ was implemented to retain the pair that gave the best pSNR and SSIM scores (e.g., $J=5, \lambda=10^{-5}$ and ISNR $\simeq 30 \mathrm{~dB}$ for Sparkling).

Importantly, we checked that both algorithms converged to the same MR image for orthogonal transforms $\left(\Psi_{\mathrm{OT}}\right)$, and we observed a slightly faster convergence for FISTA (cf Fig. 2) in this context. However, for tight frame $\Psi_{T F}$ we observed that the solution in Eq. (1) is much better than that of Eq. (2) (results not shown).

\section{MR IMAGE RECONSTRUCTION RESULTS}

\section{A. Comparison of multiscale decompositions}

We report in Fig. 3 the image quality scores (pSNR and SSIM) only for the Sparkling under-sampling scheme with

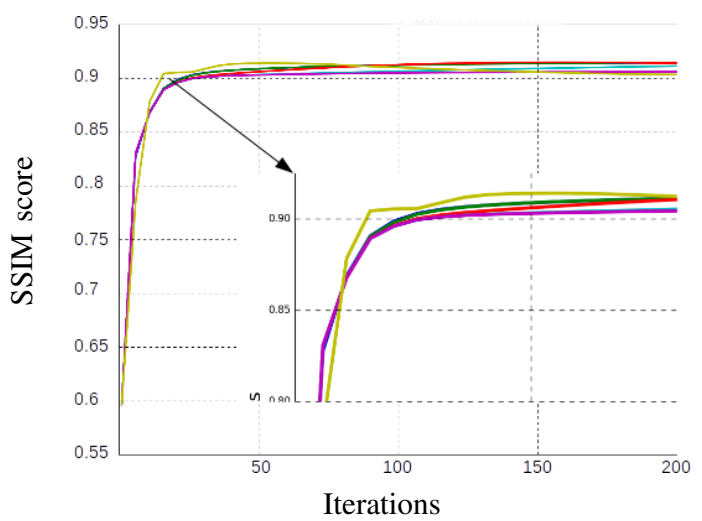

Fig. 2. Left: Evolution of the SSIM score over FISTA (yellow curve) and Condat-Vũ (blue, red and green curves) iterations for an orthogonal Mallat WT used as $\boldsymbol{\Psi}_{\mathrm{OT}}$. Insert: zoom showing that FISTA reaches the SSIM plateau slightly earlier than Condat-Vũ.

$R=2.4$ and $R=3.3$ when sweeping the different multiscale image decompositions. A similar trend was observed for the Radial scheme even though the overall image quality was lower (results not shown). Interestingly, tight frames such as fast curvelets or undecimated bi-orthogonal WT (green and blue traces, respectively) yield much better image quality scores (pSNR and SSIM) at low ISNR than orthogonal transforms depicted by yellow, red and purple curves. The worse decompositions are clearly the Mallat and B-spline WT whereas the Meyer WT provides an intermediate solution, whose computational cost is quite fast. At high ISNR, all transforms more or less converge towards the same image quality scores. As expected, we observed slightly better image quality scores for $R=2.4$ as compared to $R=3.3$.

For illustration purposes, in Fig. 4, we show the reference baboon brain image as well as all reconstructed MR images using the five tested multiscale decompositions in the scenario corresponding to an ISNR of about $30 \mathrm{~dB}$. As expected given Fig. 3, we retrieved better image quality using tight frames. It is worth noting that even though different decompositions give the same SSIM score, visually the images appear quite different. The MR image reconstructed using the Mallat WT looks noisier and the image solution reconstructed from the Meyer WT looks smoother. The same remark holds for tight frames which both achieve an SSIM score of 0.92. This basically means that any single quantitative index is not able to capture all image properties.

\section{B. Comparison of sampling schemes}

Beyond the comparison of multiscale decompositions, the under-sampling scheme may significantly impact the image quality, both in terms of shape of $k$-space trajectories (radial spokes vs Sparkling shots) and number of measurements ( $R$ factor). The current study was performed retrospectively and thus neglects potential discrepancies between prescribed sampling trajectories and the actual ones that may occur in prospective accelerated acquisition scenarios. For reconstruction, we used the undecimated bi-orthogonal WT and considered the 

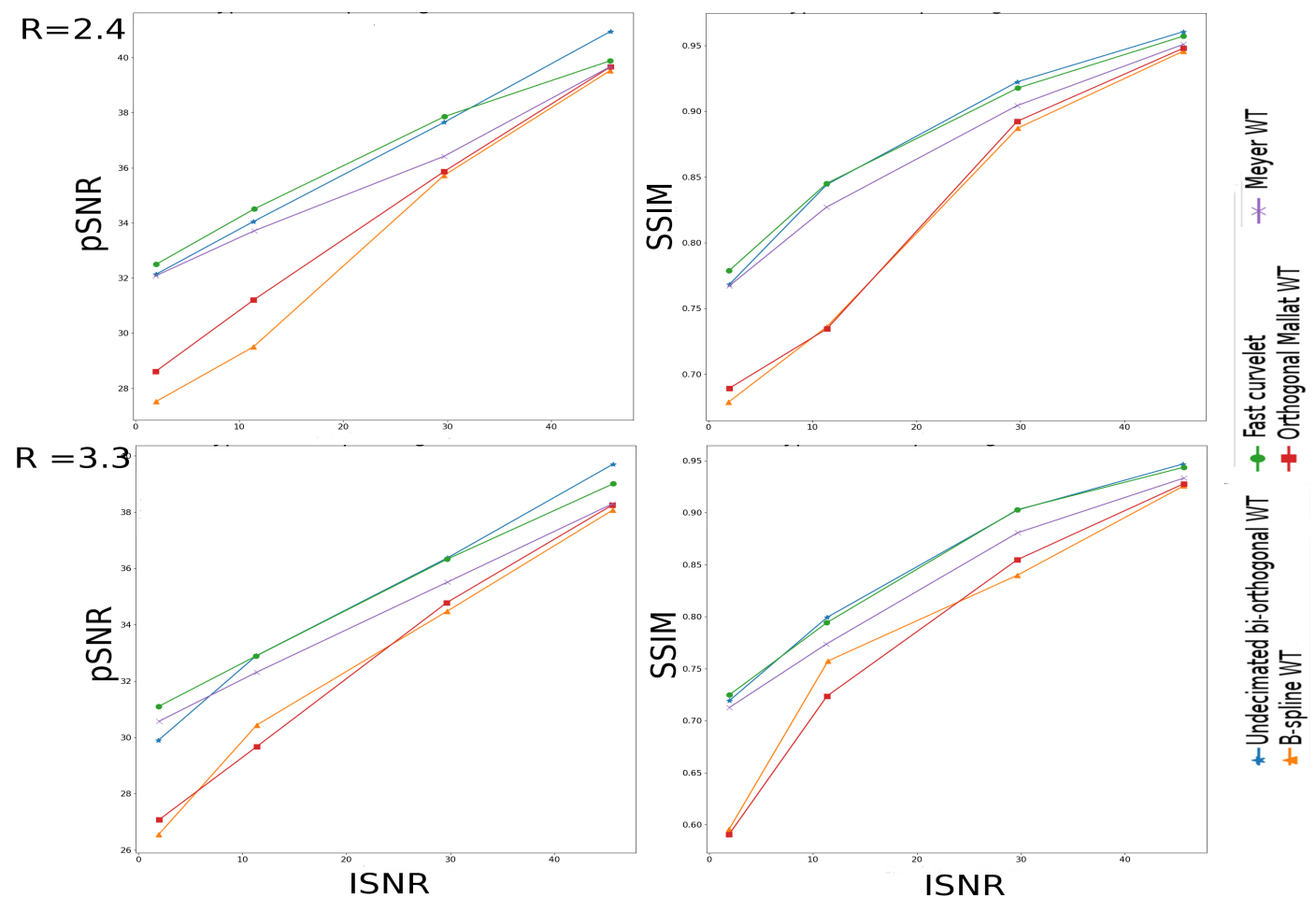

Fig. 3. pSNR (left) and SSIM (right) scores after CS-PI MR image reconstruction from retrospectively under-sampled $k$-space data using Sparkling undersampling schemes (top: $R=2.4$, bottom: $R=3.3$ ). Each line codes for a specific multiscale decomposition, see the legend within each panel.

noise-free scenario (ISNR $=40 \mathrm{~dB}$ ) to reach the asymptotic MR image quality. As shown in Fig. 5, the Sparkling pattern yielded improved MR image quality as compared to Radial lines for a given $R$ factor. Also, Sparkling is much more robust than Radial lines since it provides the same SSIM score for $R=2.4$ and $R=3.3$, whereas the image quality was significantly degraded when Radial lines were more undersampled (SSIM $=0.83$ for $R=3.3$ compared to SSIM $=0.91$ for $R=2.4$ ).

\section{Conclusions}

In this paper, we have compared analysis and synthesisbased formulations for compressed sensing MR image reconstruction in the parallel imaging context, i.e. when multiple receivers are combined within the same phased array coil. As already known in the literature, we have shown that translation invariant overcomplete decomposition outperform orthogonal wavelet transforms especially at low input SNR. Well known optimization algorithms have been respectively implemented to minimize the cost functions associated with the analysis and synthesis formulations. We have also pointed out the superiority of the Sparkling under-sampling scheme over the Radial one in terms of quality assessed by SSIM scores. We also provide a Python package, called Pysap, for CS image reconstruction in MRI and astrophysics, interfaced with the pynfft package to deal with non-Cartesian Fourier sampling.
Future work will be devoted to extend this analysis to 3D multiscale decompositions and especially to identify which of the Beam-curvelet or ridge-curvelet are more accurate to sparsify isotropic high resolution 3D MR images, which are collected using dedicated 3D Sparkling trajectories.

\section{REFERENCES}

[1] K. P. Pruessmann, M. Weiger, M. B. Scheidegger, P. Boesiger et al., "SENSE: sensitivity encoding for fast MRI," Magn. Reson. Med., vol. 42, no. 5, pp. 952-962, 1999.

[2] M. A. Griswold, P. M. Jakob, R. M. Heidemann, M. Nittka, V. Jellus, J. Wang, B. Kiefer, and A. Haase, "Generalized autocalibrating partially parallel acquisitions (GRAPPA)," Magn. Reson. Med., vol. 47, no. 6, pp. 1202-1210, 2002.

[3] D. L. Donoho, "Compressed sensing," IEEE Trans. Inf. Theory, vol. 52, no. 4, pp. 1289-1306, 2006.

[4] E. J. Candès, J. Romberg, and T. Tao, "Robust uncertainty principles: Exact signal reconstruction from highly incomplete frequency information," IEEE Trans. Inf. Theory, vol. 52, no. 2, pp. 489-509, 2006.

[5] M. Lustig, D. Donoho, and J. M. Pauly, "Sparse MRI: The application of compressed sensing for rapid MR imaging," Magn. Reson. Med., vol. 58, no. 6, pp. 1182-1195, 2007.

[6] M. Lustig, M. Alley, S. Vasanawala, D. Donoho, and J. Pauly, " $\ell_{1}$ SPIRiT: Autocalibrating parallel imaging compressed sensing," in Proc Intl Soc Mag Reson Med, vol. 17, 2009, p. 379.

[7] F. Huang, Y. Chen, W. Yin, W. Lin, X. Ye, W. Guo, and A. Reykowski, "A rapid and robust numerical algorithm for sensitivity encoding with sparsity constraints: Self-feeding sparse SENSE," Magn. Reson. Med., vol. 64, no. 4, pp. 1078-1088, 2010.

[8] M. Guerquin-Kern, M. Haberlin, K. Pruessmann, and M. Unser, "A fast wavelet-based reconstruction method for magnetic resonance imaging," IEEE Trans. Med. Imag., vol. 30, no. 9, pp. 1649-1660, 2011. 
Reference

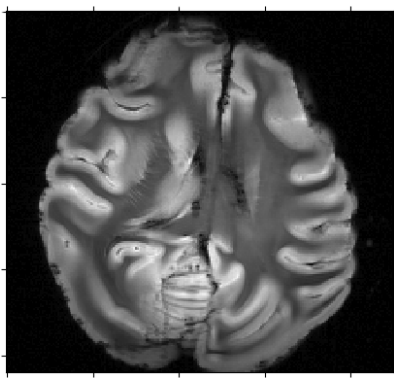

BsplineWaveletTransformATrousAlgorithm ssim: 0.89

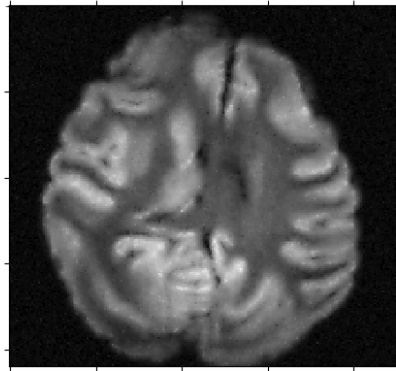

UndecimatedBiOrthogonalTransform ssim: 0.92

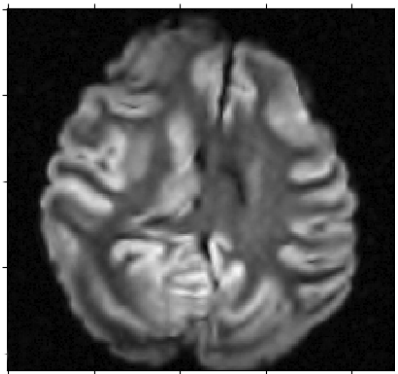

MallatWaveletTransform79Filters ssim: 0.89

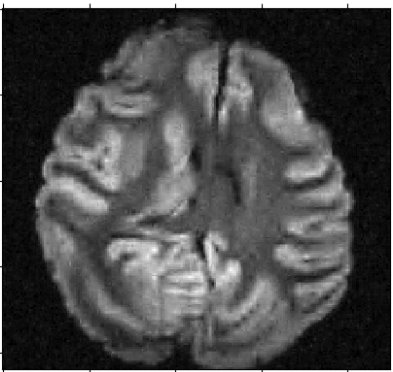

MeyerWaveletsCompactInFourierSpace ssim: 0.9

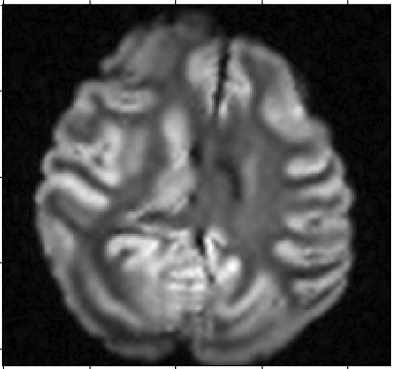

FastCurveletTransform ssim: 0.92

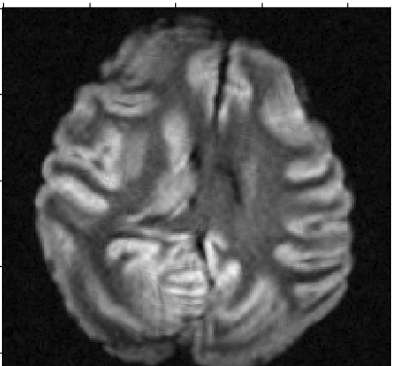

Fig. 4. Comparisons of multiscale transforms: Reconstructed MR images from retrospectively under-sampled $k$-space noisy data using Sparkling sampling pattern $(R=2.4$ and ISNR $\simeq 30 \mathrm{~dB})$. Top: Reference MR baboon image (left) and reconstructed image using Eq. (2) and Mallat WT. Center: MR images reconstructed image B-spline and Meyer WT. Bottom: MR images solutions computed using the analysis-based formulation and tight frames. The SSIM scores appear on top of each panel.

[9] L. Chaari, J.-C. Pesquet, A. Benazza-Benyahia, and P. Ciuciu, "A wavelet-based regularized reconstruction algorithm for SENSE parallel MRI with applications to neuroimaging," Med Imag. Anal., vol. 15, no. 2 , pp. 185-201, 2011.

[10] L. Chaari, P. Ciuciu, S. Mériaux, and J.-C. Pesquet, "Spatio-temporal wavelet regularization for parallel MRI reconstruction: application to functional MRI," MAGMA, vol. 27, no. 6, pp. 509-529, Dec. 2014.

[11] I. Y. Chun, B. Adcock, and T. M. Talavage, "Efficient compressed sensing SENSE pMRI reconstruction with joint sparsity promotion," IEEE Trans. Imag. Proc., vol. 35, no. 1, pp. 354-368, 2016.

[12] K. H. Jin, D. Lee, and J. C. Ye, "A general framework for compressed sensing and parallel mri using annihilating filter based low-rank Hanke matrix," IEEE Trans. Comput. Imag., vol. 2, no. 4, pp. 480-495, 2016.

[13] M. Elad, P. Milanfar, and R. Rubinstein, "Analysis versus synthesis in signal priors," Inverse problems, vol. 23, no. 3, p. 947, 2007.

[14] I. W. Selesnick and M. A. Figueiredo, "Signal restoration with overcomplete wavelet transforms: Comparison of analysis and synthesis priors," in Wavelets XIII, vol. 7446. International Society for Optics and Photonics, 2009, p. 74460D.

[15] S. Pejoski, V. Kafedziski, and D. Gleich, "Compressed sensing MRI using discrete nonseparable shearlet transform and FISTA," IEEE Sig.

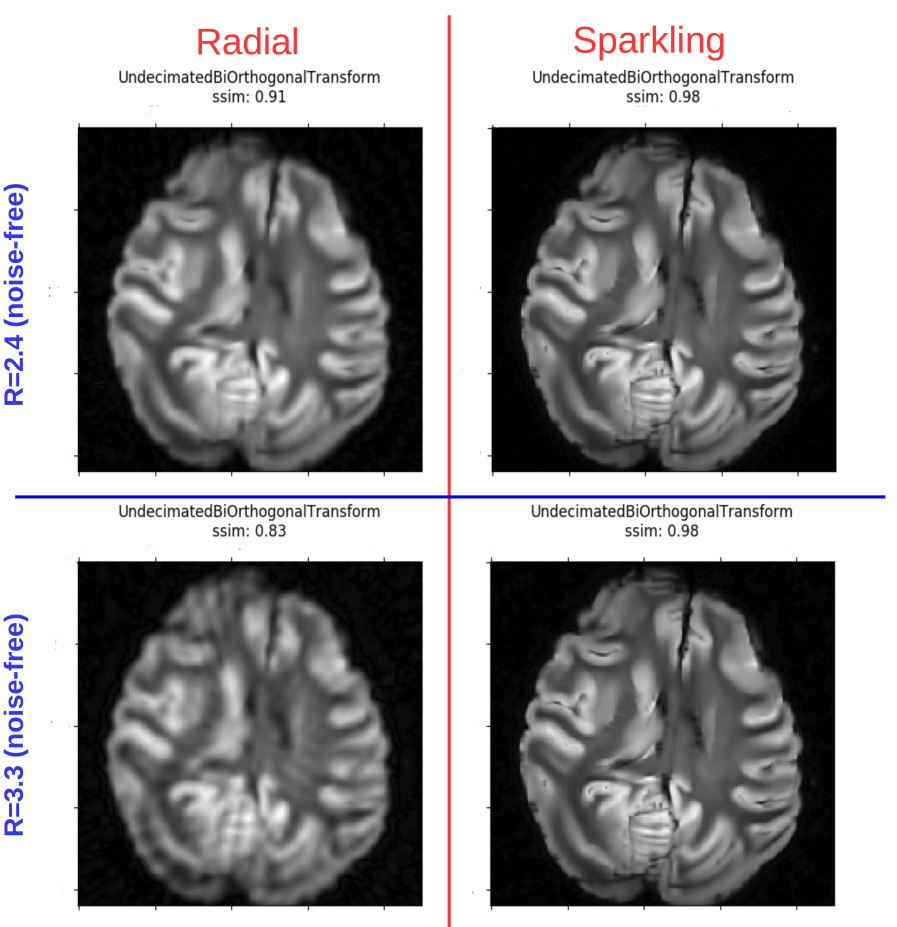

Fig. 5. Comparisons of under-sampling schemes (Radial, Sparkling) in the noise-free case. MR reconstructed images from retrospectively undersampled data $(R=2.4$ in top row, $R=3.3$ in bottom row) using the analysis-based formulation (1) with the undecimated bi-orthogonal WT.

Proc. Let., vol. 22, no. 10, pp. 1566-1570, 2015.

[16] J.-L. Starck, E. J. Candès, and D. L. Donoho, "The curvelet transform for image denoising," IEEE Trans. Imag. Proc., vol. 11, no. 6, pp. 670-684, 2002.

[17] E. Candes, L. Demanet, D. Donoho, and L. Ying, "Fast discrete curvelet transforms," Multiscale Modeling \& Simulation, vol. 5, no. 3, pp. 861899, 2006.

[18] B. C. Vũ, "A splitting algorithm for dual monotone inclusions involving cocoercive operators," Adv. in Comput. Math., vol. 38, no. 3, pp. 667681,2013

[19] L. Condat, "A primal-dual splitting method for convex optimization involving Lipschitzian, proximable and linear composite terms," J. Optim. Th. App., vol. 158, no. 2, pp. 460-479, 2013.

[20] P. L. Combettes and J.-C. Pesquet, "Proximal splitting methods in signal processing," in Fixed-point algorithms for inverse problems in science and engineering. Springer, 2011, pp. 185-212.

[21] A. Beck and M. Teboulle, "A fast iterative shrinkage-thresholding algorithm for linear inverse problems," SIAM J. Imag. Sci., vol. 2, no. 1, pp. 183-202, 2009.

[22] M. Uecker, P. Lai, M. J. Murphy, P. Virtue, M. Elad, J. M. Pauly, S. S. Vasanawala, and M. Lustig, "ESPIRiT-An Eigenvalue Approach to Autocalibrating Parallel MRI: Where SENSE Meets GRAPPA," Magn. Reson. Med., vol. 71, pp. 990-1001, 2014.

[23] L. El Gueddari, C. Lazarus, H. Carrie, A. Vignaud, and P. Ciuciu, "Self-calibrating nonlinear reconstruction algorithms for variable density sampling and parallel reception MRI," IEEE 10th Sensor Array and Multichannel Signal Processing workshop, July 2018.

[24] N. Chauffert, P. Ciuciu, and P. Weiss, "Variable density compressed sensing in MRI. Theoretical vs heuristic sampling strategies," in 10th Proc. IEEE ISBI, San Francisco, CA, Apr. 2013, pp. 298-301.

[25] C. Lazarus, P. Weiss, N. Chauffert, F. Mauconduit, M. Bottlaender, A. Vignaud, and P. Ciuciu, "SPARKLING: Novel Non-Cartesian Sampling Schemes for Accelerated 2D Anatomical Imaging at 7T Using Compressed Sensing," in 25th ISMRM conf., Honolulu, USA, 2017.

[26] C. Boyer, N. Chauffert, P. Ciuciu, J. Kahn, and P. Weiss, "On the generation of sampling schemes for magnetic resonance imaging," SIAM J. Imaging Sci., vol. 9, no. 4, pp. 2039-2072, 2016. 